Sum rul es for gener al i zed el ect r on- pai $r$ moments of Hartree Fock or bi tal s

\begin{tabular}{|l|l|}
\hline 著者 & KOGA Toshi kat su \\
\hline $\begin{array}{l}\text { j our nal or } \\
\text { publ i cat i on t i t l e }\end{array}$ & The $\mathrm{j}$ our nal of chemi cal physi cs \\
\hline vol une & 114 \\
\hline number & 19 \\
\hline page $\mathrm{r}$ ange & $8382-8385$ \\
\hline year & 2001- 05- 15 \\
\hline URL & ht t p: //hdl . handl e. net /10258/792 \\
\hline
\end{tabular}




\title{
Sum rules for generalized electron-pair moments of Hartree-Fock orbitals
}

\author{
Toshikatsu Koga \\ Department of Applied Chemistry, Muroran Institute of Technology, Muroran, Hokkaido 050-8585, Japan
}

(Received 17 January 2001; accepted 2 March 2001)

For many-electron systems, the generalized electron-pair density function $g(q ; a, b)$ represents the probability density function for the magnitude $\left|a \mathbf{r}_{i}+b \mathbf{r}_{j}\right|$ of two-electron vector $a \mathbf{r}_{i}+b \mathbf{r}_{j}$ to be $q$, where $a$ and $b$ are real-valued parameters. For Hartree-Fock wave functions, decomposition of $g(q ; a, b)$ into spin-orbital-pair components $g^{i j}(q ; a, b)$ shows that the second moments $\left\langle q^{2}\right\rangle_{(a, b)}^{i j}$, associated with $g^{i j}(q ; a, b)$, satisfy several rigorous sum rules which connect one- and two-electron properties of spin-orbitals $i$ and $j$. The same is also true in momentum space. As an illustrative application, the orbital kinetic energies of the $\mathrm{Rn}$ atom are separated into the relative motion and center-of-mass motion contributions of electrons in two relevant orbitals. (c) 2001 American Institute of Physics. [DOI: 10.1063/1.1367371]

\section{INTRODUCTION}

In recent papers, we have studied ${ }^{1,2}$ a generalized electron-pair density function $g(q ; a, b)$ defined by

$$
g(q ; a, b) \equiv\left(4 \pi q^{2}\right)^{-1}\left\langle\sum_{i=1}^{N-1} \sum_{j=i+1}^{N} \delta\left(q-\left|a \mathbf{r}_{i}+b \mathbf{r}_{j}\right|\right)\right\rangle,
$$

where $a$ and $b$ are real-valued parameters, $\delta(x)$ is the onedimensional Dirac delta function, and the angular brackets \langle\rangle stand for the expectation value over the $N$-electron $(N \geqslant 2)$ wave function $\Psi\left(\mathbf{x}_{1}, \ldots, \mathbf{x}_{N}\right)$ with $\mathbf{x}_{i} \equiv\left(\mathbf{r}_{i}, \sigma_{i}\right)$ being the combined position-spin coordinates of the electron $i$. The generalized electron-pair density $g(q ; a, b)$ represents the probability density function for the magnitude $\left|a \mathbf{r}_{i}+b \mathbf{r}_{j}\right|$ of twoelectron vector $a \mathbf{r}_{i}+b \mathbf{r}_{j}$ of any pair of electrons $i$ and $j$ to be $q$, and is normalized to $N(N-1) / 2$, the number of electron pairs. It has been shown ${ }^{2}$ that the function $g(q ; a, b)$ connects smoothly the single-electron density ${ }^{3} \rho(r)$, the electron-pair intracule (relative motion) density ${ }^{4-8} h(u)$, and the electron-pair extracule (center-of-mass motion) density $^{4-8} d(R)$. Namely,

$$
\begin{aligned}
& g(q ; 1,-1)=h(q), \quad g(q ; 1,0)=\frac{N-1}{2} \rho(q), \\
& g(q ; 1,+1)=\frac{1}{8} d\left(\frac{q}{2}\right) .
\end{aligned}
$$
by

If we define moments $\left\langle q^{k}\right\rangle_{(a, b)}$ of the density $g(q ; a, b)$

$$
\begin{aligned}
\left\langle q^{k}\right\rangle_{(a, b)} & \equiv 4 \pi \int_{0}^{\infty} d q q^{k+2} g(q ; a, b) \\
& =\left\langle\sum_{i=1}^{N-1} \sum_{j=i+1}^{N}\left|a \mathbf{r}_{i}+b \mathbf{r}_{j}\right|^{k}\right\rangle,
\end{aligned}
$$

we then find

$$
\begin{aligned}
& \left\langle q^{k}\right\rangle_{(1,-1)}=\left\langle u^{k}\right\rangle, \quad\left\langle q^{k}\right\rangle_{(1,0)}=\frac{N-1}{2}\left\langle r^{k}\right\rangle, \\
& \left\langle q^{k}\right\rangle_{(1,+1)}=2^{k}\left\langle R^{k}\right\rangle,
\end{aligned}
$$

corresponding to Eq. (2), and all the single-electron $\left\langle r^{k}\right\rangle$, intracule $\left\langle u^{k}\right\rangle$, and extracule $\left\langle R^{k}\right\rangle$ moments are generated from the generalized electron-pair moments $\left\langle q^{k}\right\rangle_{(a, b)}$. In addition, it has been found ${ }^{9}$ that there exists a rigorous sum rule for the second generalized electron-pair moments $\left\langle q^{2}\right\rangle_{(a, b)}$ :

$$
\left\langle q^{2}\right\rangle_{(a, b)}+\left\langle q^{2}\right\rangle_{(a,-b)}=\left(a^{2}+b^{2}\right)(N-1)\left\langle r^{2}\right\rangle,
$$

which is valid for both exact and approximate wave functions of any atoms and molecules. It is interesting that the right-hand side of Eq. (5) is a single-electron property, while the left-hand side is a two-electron property if $a \neq 0$ and $b$ $\neq 0$. For a special case of $a=b=1$, Eq. (5) reads

$$
\left\langle u^{2}\right\rangle+4\left\langle R^{2}\right\rangle=2(N-1)\left\langle r^{2}\right\rangle .
$$

Namely, the sum of the second intracule $\left\langle u^{2}\right\rangle$ and extracule $\left\langle R^{2}\right\rangle$ moments (the latter multiplied by 4 ) is exactly identical with the second single-electron moment $\left\langle r^{2}\right\rangle$ multiplied by $2(N-1)$.

When we introduce the corresponding electron-pair density $\bar{g}(t ; a, b)$ and associated moments $\left\langle t^{n}\right\rangle_{(a, b)}$ in momentum space, exactly the same discussion as in position space results ${ }^{9}$ in the momentum-space counterparts of Eqs. (5) and (6).

In the present paper, we discuss decomposition of the generalized electron-pair density $g(q ; a, b)$ and its second moment $\left\langle q^{2}\right\rangle_{(a, b)}$ into spin-orbital-pair contributions $g^{i j}(q ; a, b)$ and $\left\langle q^{2}\right\rangle_{(a, b)}^{i j}$, respectively, within the HartreeFock framework. Several sum rules are derived which connect one- and two-electron properties associated with spinorbitals $i$ and $j$. For atomic systems, sum rules for subshell contributions are also obtained. The isomorphism of determinantal wave functions in position and momentum spaces leads that exactly the same sum rules hold in momentum space as well. An application of the present results is illus- 
trated for the separation of the orbital kinetic energies of the $\mathrm{Rn}$ atom into the relative motion and center-of-mass motion contributions of electrons in two relevant orbitals. Hartree atomic units are used throughout.

\section{SUM RULES FOR HARTREE-FOCK ORBITALS}

\section{A. Position space}

For an $\mathrm{N}$-electron determinantal wave function composed of a set of orthonormal spin-orbitals $\psi_{i}(\mathbf{r}) \eta_{i}(\sigma)$, the electron-pair density $g(q ; a, b)$, defined by Eq. (1), is rewritten as

$$
\begin{aligned}
g(q ; a, b)= & \sum_{i=1}^{N-1} \sum_{j=i+1}^{N} g^{i j}(q ; a, b), \\
g^{i j}(q ; a, b)= & \left(4 \pi q^{2}\right)^{-1}\left\langle\psi_{i}\left(\mathbf{r}_{1}\right) \psi_{j}\left(\mathbf{r}_{2}\right)\right| \frac{1}{2}\left[\delta\left(q-\left|a \mathbf{r}_{1}+b \mathbf{r}_{2}\right|\right)\right. \\
& \left.+\delta\left(q-\left|b \mathbf{r}_{1}+a \mathbf{r}_{2}\right|\right)\right] \\
& \times\left|\psi_{i}\left(\mathbf{r}_{1}\right) \psi_{j}\left(\mathbf{r}_{2}\right)-\delta_{s}(i, j) \psi_{j}\left(\mathbf{r}_{1}\right) \psi_{i}\left(\mathbf{r}_{2}\right)\right\rangle
\end{aligned}
$$

where the angular brackets abbreviate the integrations over $\mathbf{r}_{1}$ and $\mathbf{r}_{2}$, and the spin integral $\delta_{s}(i, j)$ is unity if the spinorbitals $i$ and $j$ have the same spin and is zero if they have the opposite spins. Corresponding to Eqs. (7a) and (7b), the generalized electron-pair moments $\left\langle q^{k}\right\rangle_{(a, b)}$ are decomposed as

$$
\begin{aligned}
\left\langle q^{k}\right\rangle_{(a, b)}= & \sum_{i=1}^{N-1} \sum_{j=i+1}^{N}\left\langle q^{k}\right\rangle_{(a, b)}^{i j}, \\
\left\langle q^{k}\right\rangle_{(a, b)}^{i j} \equiv & 4 \pi \int_{0}^{\infty} d q q^{k+2} g^{i j}(q ; a, b) \\
= & \left\langle\psi_{i}\left(\mathbf{r}_{1}\right) \psi_{j}\left(\mathbf{r}_{2}\right)\right| \frac{1}{2}\left[\left|a \mathbf{r}_{1}+b \mathbf{r}_{2}\right|^{k}+\left|b \mathbf{r}_{1}+a \mathbf{r}_{2}\right|^{k}\right] \\
& \times\left|\psi_{i}\left(\mathbf{r}_{1}\right) \psi_{j}\left(\mathbf{r}_{2}\right)-\delta_{s}(i, j) \psi_{j}\left(\mathbf{r}_{1}\right) \psi_{i}\left(\mathbf{r}_{2}\right)\right\rangle .
\end{aligned}
$$

For a particular case of $k=2$, the moment operator in Eq. (8b) satisfies an identity

$$
\begin{aligned}
& \frac{1}{2}\left[\left|a \mathbf{r}_{1}+b \mathbf{r}_{2}\right|^{2}+\left|b \mathbf{r}_{1}+a \mathbf{r}_{2}\right|^{2}\right] \\
& \quad=\frac{1}{2}\left(a^{2}+b^{2}\right)\left(r_{1}^{2}+r_{2}^{2}\right)+2 a b \mathbf{r}_{1} \cdot \mathbf{r}_{2},
\end{aligned}
$$

where $r_{i}=\left|\mathbf{r}_{i}\right|$. We then find from Eq. (8b) that

$$
\begin{aligned}
\left\langle q^{2}\right\rangle_{(a, b)}^{i j}= & \frac{1}{2}\left(a^{2}+b^{2}\right)\left[\left\langle r^{2}\right\rangle_{i}+\left\langle r^{2}\right\rangle_{j}\right] \\
& +2 a b\left[\langle\mathbf{r}\rangle_{i i} \cdot\langle\mathbf{r}\rangle_{j j}-\delta_{s}(i, j)\left|\langle\mathbf{r}\rangle_{i j}\right|^{2}\right],
\end{aligned}
$$

where

$$
\langle\mathbf{r}\rangle_{i j}=\int d \mathbf{r} \psi_{i}^{*}(\mathbf{r}) \mathbf{r} \psi_{j}(\mathbf{r}) .
$$

Therefore, Eqs. (10) for $\left\langle q^{2}\right\rangle_{(a, b)}^{i j}$ and $\left\langle q^{2}\right\rangle_{(a,-b)}^{i j}$ generate a rigorous sum rule

$$
\left\langle q^{2}\right\rangle_{(a, b)}^{i j}+\left\langle q^{2}\right\rangle_{(a,-b)}^{i j}=\left(a^{2}+b^{2}\right)\left[\left\langle r^{2}\right\rangle_{i}+\left\langle r^{2}\right\rangle_{j}\right],
$$

for the second generalized electron-pair moments associated with the spin-orbitals $i$ and $j$. Summation of Eq. (11) over $1 \leqslant i<j \leqslant N$ results in Eq. (5). For any values of the parameters $a$ and $b$ and any pairs of spin-orbitals $i$ and $j$, Eq. (11) shows that the sum of two symmetric electron-pair moments $\left\langle q^{2}\right\rangle_{(a, b)}^{i j}$ and $\left\langle q^{2}\right\rangle_{(a,-b)}^{i j}$ is equal to a single-electron property $\left\langle r^{2}\right\rangle_{i}+\left\langle r^{2}\right\rangle_{j}$ multiplied by $a^{2}+b^{2}$. For any two sets of parameters $(a, b)$ and $\left(a^{\prime}, b^{\prime}\right)$, Eq. (11) also gives

$$
\frac{\left\langle q^{2}\right\rangle_{(a, b)}^{i j}+\left\langle q^{2}\right\rangle_{(a,-b)}^{i j}}{a^{2}+b^{2}}=\frac{\left\langle q^{2}\right\rangle_{\left(a^{\prime}, b^{\prime}\right)}^{i j}+\left\langle q^{2}\right\rangle_{\left(a^{\prime},-b^{\prime}\right)}^{i j}}{a^{\prime 2}+b^{\prime 2}} .
$$

Note that Eqs. (11) and (12) are valid for both exact and approximate Hartree-Fock wave functions, since they originate from the operator identity, Eq. (9), not from a particular property of spin-orbitals.

For a special case of $a=b=1$, Eq. (11) reads

$$
\left\langle u^{2}\right\rangle^{i j}+4\left\langle R^{2}\right\rangle^{i j}=2\left[\left\langle r^{2}\right\rangle_{i}+\left\langle r^{2}\right\rangle_{j}\right] \text {. }
$$

Namely, the sum of the second intracule $\left\langle u^{2}\right\rangle^{i j}$ and extracule $\left\langle R^{2}\right\rangle^{i j}$ moments (the latter multiplied by 4 ), associated with the spin-orbitals $i$ and $j$, is exactly twice the sum of the second single-electron moments $\left\langle r^{2}\right\rangle_{i}$ and $\left\langle r^{2}\right\rangle_{j}$ of the relevant two spin-orbitals. Equation (13) enables us to separate the sum of orbital single-electron moments into the relative motion and center-of-mass motion contributions in a precise manner.

When the system under consideration has spatial inversion symmetry, we can also derive a direct relation between $\left\langle q^{2}\right\rangle_{(a, b)}^{i j}$, instead of the symmetric sum, and single-electron orbital moments. If the spatial function $\psi_{i}(\mathbf{r})$ has inversion symmetry $\psi_{i}(-\mathbf{r})=(-1)^{\lambda_{i}} \psi_{i}(\mathbf{r})$ specified by an index $\lambda_{i}$, the first term $\langle\mathbf{r}\rangle_{i i} \cdot\langle\mathbf{r}\rangle_{j j}$ in the brackets after the coefficient $2 a b$ in Eq. (10a) vanishes. The second term $\delta_{s}(i, j)\left|\langle\mathbf{r}\rangle_{i j}\right|^{2}$ is also zero, if the spin-orbitals $i$ and $j$ have different spins or their spatial inversion symmetries are the same, $\psi_{i}(-\mathbf{r})$ $\times \psi_{j}(-\mathbf{r})=\psi_{i}(\mathbf{r}) \psi_{j}(\mathbf{r})$. In this particular case, Eq. (10a) is simplified to

$$
\left\langle q^{2}\right\rangle_{(a, b)}^{i j}=\frac{1}{2}\left(a^{2}+b^{2}\right)\left[\left\langle r^{2}\right\rangle_{i}+\left\langle r^{2}\right\rangle_{j}\right],
$$

which further yields

$$
\left\langle u^{2}\right\rangle^{i j}=4\left\langle R^{2}\right\rangle^{i j}=\left\langle r^{2}\right\rangle_{i}+\left\langle r^{2}\right\rangle_{j},
$$

for the spin-orbital-pair intracule and extracule moments when $a=1$ and $b= \pm 1$. The relative motion and center-ofmass motion contributions in Eq. (13) are the same in this case. When the spin-orbitals $i$ and $j$ belong to the same subshell $n l$ of an atom, where $n$ and $l$ are the principal and azimuthal quantum numbers, Eqs. (14a) and (14b) always hold as well as an equality $\left\langle r^{2}\right\rangle_{i}=\left\langle r^{2}\right\rangle_{j}$. We then have

$$
\begin{gathered}
\left\langle q^{2}\right\rangle_{(a, b)}^{n l}=\frac{1}{2}\left(a^{2}+b^{2}\right)\left(N_{n l}-1\right)\left\langle r^{2}\right\rangle_{n l}, \\
\left\langle u^{2}\right\rangle^{n l}=4\left\langle R^{2}\right\rangle^{n l}=\left(N_{n l}-1\right)\left\langle r^{2}\right\rangle_{n l},
\end{gathered}
$$

where our subshell normalization is $\left\langle q^{0}\right\rangle_{(a, b)}^{n l}=N_{n l}\left(N_{n l}\right.$ $-1) / 2$ and $\left\langle r^{0}\right\rangle_{n l}=N_{n l}$, in which $N_{n l}(\geqslant 2)$ is the number of electrons in the subshell $n l$. Equation (15b) was also reported in Ref. 10.

\section{B. Momentum space}

The Hartree-Fock wave function in momentum space has exactly the same determinantal structure as in position space, provided the single-electron spatial function $\psi_{i}(\mathbf{r})$ is replaced with 


$$
\phi_{i}(\mathbf{p})=(2 \pi)^{-3 / 2} \int d \mathbf{r} \exp (-i \mathbf{p} \cdot \mathbf{r}) \psi_{i}(\mathbf{r}) .
$$

Then the spin-orbital-pair components $\bar{g}^{i j}(t ; a, b)$ and $\left\langle t^{k}\right\rangle_{(a, b)}^{i j}$ of the generalized electron-pair density $\bar{g}(t ; a, b)$ and moments $\left\langle t^{k}\right\rangle_{(a, b)}$ in momentum space satisfy various relations discussed above in position space, if the variables and functions are replaced appropriately. An exception is that the orbital density $\Pi_{i}(\mathbf{p}) \equiv\left|\phi_{i}(\mathbf{p})\right|^{2}$ in momentum space has even inversion symmetry $\Pi_{i}(-\mathbf{p})=\Pi_{i}(\mathbf{p})$ for any atoms and molecules. Therefore, the $\langle\mathbf{p}\rangle_{i i} \cdot\langle\mathbf{p}\rangle_{j j}$ term is absent in the momentum-space counterpart of Eq. (10a).

Some explicit forms of the spin-orbital-pair sum rules in momentum space are

$$
\begin{aligned}
& \left\langle t^{2}\right\rangle_{(a, b)}^{i j}+\left\langle t^{2}\right\rangle_{(a,-b)}^{i j}=\left(a^{2}+b^{2}\right)\left[\left\langle p^{2}\right\rangle_{i}+\left\langle p^{2}\right\rangle_{j}\right], \\
& \left\langle v^{2}\right\rangle^{i j}+4\left\langle P^{2}\right\rangle^{i j}=2\left[\left\langle p^{2}\right\rangle_{i}+\left\langle p^{2}\right\rangle_{j}\right],
\end{aligned}
$$

for any two spin-orbitals, and

$$
\begin{aligned}
& \left\langle t^{2}\right\rangle_{(a, b)}^{i j}=\frac{1}{2}\left(a^{2}+b^{2}\right)\left[\left\langle p^{2}\right\rangle_{i}+\left\langle p^{2}\right\rangle_{j}\right], \\
& \left\langle v^{2}\right\rangle^{i j}=4\left\langle P^{2}\right\rangle^{i j}=\left\langle p^{2}\right\rangle_{i}+\left\langle p^{2}\right\rangle_{j},
\end{aligned}
$$

for two spin-orbitals with the same inversion symmetry, where $p, v$, and $P$ are the momentum-space single-electron, intracule, and extracule radii, respectively. Even when two spin-orbitals have different inversion symmetries, Eqs. (18a) and (18b) are valid for Hartree wave functions where there are no exchange terms. Equation (17) is of our special interest, since the second single-electron moment $\left\langle p^{2}\right\rangle_{i}$ appearing on the right-hand side is just twice the electronic kinetic energy $T_{i}=\left\langle p^{2}\right\rangle_{i} / 2$ of the spin-orbital $i$. When Eq. (17b) is applied, the sum $T_{i}+T_{j}$ of the orbital kinetic energies is precisely decomposed into two contributions from the relative (intracule) and center-of-mass (extracule) motions of an electron pair. Some numerical results will be presented in the next section.

We note that comparison of Eqs. (11) and (17a) gives an additional rigorous relation

$$
\begin{aligned}
& {\left[\left\langle p^{2}\right\rangle_{i}+\left\langle p^{2}\right\rangle_{j}\right]\left[\left\langle q^{2}\right\rangle_{(a, b)}^{i j}+\left\langle q^{2}\right\rangle_{(a,-b)}^{i j}\right]} \\
& =\left[\left\langle r^{2}\right\rangle_{i}+\left\langle r^{2}\right\rangle_{j}\right]\left[\left\langle t^{2}\right\rangle_{(a, b)}^{i j}+\left\langle t^{2}\right\rangle_{(a,-b)}^{i j}\right],
\end{aligned}
$$

which connects one- and two-electron moments in position and momentum spaces. If we consider all the spin-orbitals $i$ and $j$ belonging to the same subshell $n l$ of an atom, Eq. (19a) is simplified to

$$
\left\langle p^{2}\right\rangle_{n l}\left\langle q^{2}\right\rangle_{(a, b)}^{n l}=\left\langle r^{2}\right\rangle_{n l}\left\langle t^{2}\right\rangle_{(a, b)}^{n l},
$$

for any values of $a$ and $b$.

\section{AN ILLUSTRATIVE APPLICATION}

According to Eq. (17b), the sum of two orbital kinetic energies is exactly decomposed into the intracule and extracule contributions as

$$
T_{i}+T_{j}=T_{\mathrm{int}}^{i j}+T_{\mathrm{ext}}^{i j},
$$

where

$$
T_{\mathrm{int}}^{i j}=\frac{1}{4}\left\langle v^{2}\right\rangle^{i j}, \quad T_{\mathrm{ext}}^{i j}=\left\langle P^{2}\right\rangle^{i j} .
$$

TABLE I. Ratios between the relative motion and center-of-mass motion contributions in the orbital kinetic energies of the $\mathrm{Rn}$ atom. The magnetic and spin quantum numbers are not specified for the spin-orbitals, since any allowed values give the identical result.

\begin{tabular}{rccccccc}
\hline \hline No. & $i$ & $j$ & $T_{\text {int }}^{i j} / T_{\mathrm{ext}}^{i j}$ & No. & $i$ & $j$ & $T_{\mathrm{int}}^{i j} / T_{\mathrm{ext}}^{i j}$ \\
\hline 1 & $1 s$ & $2 p$ & 1.12029 & 28 & $3 p$ & $5 s$ & 1.00268 \\
2 & $2 p$ & $3 d$ & 1.06583 & 29 & $5 s$ & $6 p$ & 1.00222 \\
3 & $2 s$ & $3 p$ & 1.06322 & 30 & $3 d$ & $4 p$ & 1.00207 \\
4 & $3 s$ & $4 p$ & 1.03353 & 31 & $4 p$ & $6 s$ & 1.00193 \\
5 & $3 d$ & $4 f$ & 1.03168 & 32 & $2 p$ & $5 d$ & 1.00193 \\
6 & $1 s$ & $3 p$ & 1.02348 & 33 & $2 p$ & $4 s$ & 1.00189 \\
7 & $3 p$ & $4 d$ & 1.02300 & 34 & $3 s$ & $3 p$ & 1.00165 \\
8 & $6 s$ & $6 p$ & 1.02139 & 35 & $3 p$ & $3 d$ & 1.00164 \\
9 & $5 p$ & $5 d$ & 1.01817 & 36 & $4 s$ & $6 p$ & 1.00164 \\
10 & $2 s$ & $4 p$ & 1.01591 & 37 & $1 s$ & $5 p$ & 1.00115 \\
11 & $4 p$ & $5 s$ & 1.01408 & 38 & $4 f$ & $5 d$ & 1.00110 \\
12 & $4 s$ & $5 p$ & 1.01326 & 39 & $3 s$ & $6 p$ & 1.00081 \\
13 & $3 p$ & $4 s$ & 1.01268 & 40 & $2 p$ & $5 s$ & 1.00042 \\
14 & $2 p$ & $4 d$ & 1.01261 & 41 & $4 d$ & $6 p$ & 1.00041 \\
15 & $5 p$ & $6 s$ & 1.01214 & 42 & $3 p$ & $6 s$ & 1.00038 \\
16 & $5 s$ & $5 p$ & 1.01144 & 43 & $3 d$ & $5 p$ & 1.00037 \\
17 & $4 d$ & $4 f$ & 1.00854 & 44 & $2 s$ & $6 p$ & 1.00035 \\
18 & $2 p$ & $3 s$ & 1.00773 & 45 & $2 s$ & $2 p$ & 1.00033 \\
19 & $3 s$ & $5 p$ & 1.00736 & 46 & $1 s$ & $6 p$ & 1.00012 \\
20 & $4 p$ & $4 d$ & 1.00694 & 47 & $2 p$ & $6 s$ & 1.00006 \\
21 & $1 s$ & $4 p$ & 1.00578 & 48 & $3 d$ & $6 p$ & 1.00004 \\
22 & $4 s$ & $4 p$ & 1.00492 & 49 & $1 s$ & $4 f$ & 1.00000 \\
23 & $4 d$ & $5 p$ & 1.00406 & 50 & $2 s$ & $4 f$ & 1.00000 \\
24 & $5 d$ & $6 p$ & 1.00400 & 51 & $3 s$ & $4 f$ & 1.00000 \\
25 & $3 p$ & $5 d$ & 1.00396 & 52 & $4 s$ & $4 f$ & 1.00000 \\
26 & $4 p$ & $5 d$ & 1.00394 & 53 & $4 f$ & $5 s$ & 1.00000 \\
27 & $2 s$ & $5 p$ & 1.00328 & 54 & $4 f$ & $6 s$ & 1.00000 \\
\hline \hline
\end{tabular}

If the Hartree orbitals are considered or if the Hartree-Fock orbitals $i$ and $j$ have the same inversion symmetry in momentum space, $\phi_{i}(-\mathbf{p}) \phi_{j}(-\mathbf{p})=\phi_{i}(\mathbf{p}) \phi_{j}(\mathbf{p})$, then we have

$$
T_{\mathrm{int}}^{i j}=T_{\mathrm{ext}}^{i j}=\frac{1}{2}\left(T_{i}+T_{j}\right),
$$

from Eq. (18b) and the intracule contribution is equal to the extracule one. In atomic systems, Eq. (20c) holds when the sum $l_{i}+l_{j}$ of the azimuthal quantum numbers of two Hartree-Fock spin-orbitals is even.

We have examined the intracule and extracule contributions in the orbital kinetic energies for the $\mathrm{Rn}$ atom (atomic number 86), in which 15 subshells, $1 s$ to $6 s, 2 p$ to $6 p, 3 d$ to $5 d$, and $4 f$, are fully occupied. We have 3655 electron pairs in total, but the associated orbital kinetic energy pairs are classified into 120 different cases specified by two subshells $n l$ and $n^{\prime} l^{\prime}$. Among them, 66 subshell pairs with $l$ $+l^{\prime}=$ even have $T_{\mathrm{int}}^{i j} / T_{\mathrm{ext}}^{i j}=1$ due to Eq. (20c). The 1807 electron pairs in this category thus have exactly the same kinetic energy contributions from the relative and center-ofmass motions. For the remaining 54 subshell pairs with $l$ $+l^{\prime}=$ odd (which include 1848 electron pairs), we have obtained the ratio $T_{\text {int }}^{i j} / T_{\text {ext }}^{i j}$ from numerical Hartree-Fock calculations. The results are summarized in Table $I$ in the descending order of the $T_{\mathrm{int}}^{i j} / T_{\mathrm{ext}}^{i j}$ values. We find in the table that $T_{\text {int }}^{i j} / T_{\text {ext }}^{i j} \geqslant 1$ with no exceptions, which implies that the relative motion contribution is never smaller than the centerof-mass motion contribution in any pairs of the orbital kinetic energies. Though there are many exceptions, the ratio $T_{\text {int }}^{i j} / T_{\text {ext }}^{i j}$ is generally larger when $\left|l-l^{\prime}\right|=1$ and $\left|n-n^{\prime}\right|$ is 
small. The largest value is 1.12029 for the $1 s 2 p$ electron pairs, and the intracule contribution occupies $52.8 \%$ of the orbital kinetic energy sum $T_{1 s}+T_{2 p}$. The exchange effect, appeared in the orbital kinetic energies, is largest for these electron pairs. On the other hand, $T_{\text {int }}^{i j} / T_{\text {ext }}^{i j}$ is essentially unity for the $s f$ electron pairs with $\left|l-l^{\prime}\right|=3$. When summed over all $1 \leqslant i<j \leqslant N$, the ratio $T_{\text {int }} / T_{\text {ext }}$ between the total intracule $T_{\text {int }}$ and extracule $T_{\text {ext }}$ contributions is 1.00891 .

\section{SUMMARY}

For Hartree-Fock wave functions, the generalized electron-pair density function $g(q ; a, b)$ has been decomposed into spin-orbital-pair components $g^{i j}(q ; a, b)$. Then the second moments $\left\langle q^{2}\right\rangle_{(a, b)}^{i j}$, associated with $g^{i j}(q ; a, b)$, have been shown to satisfy several rigorous sum rules which connect one- and two-electron properties of spin-orbitals $i$ and $j$. The same is also true in momentum space. An appli- cation of the present results has been illustrated for the relative motion and center-of-mass motion decomposition of the orbital kinetic energies of the $\mathrm{Rn}$ atom.

${ }^{1}$ T. Koga, Theor. Chem. Acc. 105, 96 (2000).

${ }^{2}$ T. Koga, J. Chem. Phys. 114, 72 (2001); Erratum (in press).

${ }^{3}$ P.-O. Löwdin, Phys. Rev. 97, 1474 (1955).

${ }^{4}$ A. J. Coleman, Int. J. Quantum Chem., Symp. 1, 457 (1967).

${ }^{5}$ A. J. Thakkar, in Density Matrices and Density Functionals, edited by R. M. Erdahl and V. H. Smith, Jr. (Reidel, Dordrecht, 1987), pp. 553-581.

${ }^{6}$ R. J. Boyd and J. M. Ugalde, in Computational Chemistry, Part A, edited by S. Fraga (Elsevier, Amsterdam, 1992), pp. 273-299.

${ }^{7}$ E. Valderrama, J. M. Ugalde, and R. J. Boyd, in Many-Electron Densities and Reduced Density Matrices, edited by J. Cioslowski (Kluwer Academic/Plenum, New York, 2000), pp. 231-248.

${ }^{8}$ T. Koga, in Many-Electron Densities and Reduced Density Matrices, edited by J. Cioslowski (Kluwer Academic/Plenum, New York, 2000), pp. 267-298.

${ }^{9}$ T. Koga, J. Chem. Phys. 114, 2511 (2001).

${ }^{10}$ T. Koga and H. Matsuyama, Theor. Chem. Acc. 99, 320 (1998). 